\title{
Patterns arising from the interaction between scalar and vectorial instabilities in two-photon resonant Kerr cavities
}

\author{
Miguel Hoyuelos, ${ }^{1,2, *}$ Daniel Walgraef, ${ }^{1, *, \dagger}$ Pere Colet,,${ }^{1, *}$ and Maxi San Miguel ${ }^{1, *}$ \\ ${ }^{1}$ Instituto Mediterráneo de Estudios Avanzados, IMEDEA (CSIC-UIB), Campus Universitat Illes Balears, \\ E-07071 Palma de Mallorca, Spain \\ ${ }^{2}$ Departamento de Física, Facultad de Ciencias Exactas y Naturales, Universidad Nacional de Mar del Plata, Funes 3350 , \\ 7600 Mar del Plata, Argentina
}

(Received 14 December 2001; published 11 April 2002)

\begin{abstract}
We study pattern formation associated with the polarization degree of freedom of the electric field amplitude in a mean field model describing a nonlinear Kerr medium close to a two-photon resonance, placed inside a ring cavity with flat mirrors and driven by a coherent $\hat{x}$-polarized plane-wave field. In the self-focusing case, for negative detunings the pattern arises naturally from a codimension two bifurcation. For a critical value of the field intensity there are two wave numbers that become unstable simultaneously, corresponding to two Turing-like instabilities. Considered alone, one of the instabilities would originate a linearly polarized hexagonal pattern whereas the other instability is of pure vectorial origin and would give rise to an elliptically polarized stripe pattern. We show that the competition between the two wave numbers can originate different structures, the detuning being a natural selection parameter.
\end{abstract}

DOI: $10.1103 /$ PhysRevE.65.046620

PACS number(s): 42.65.Sf, 47.54.+r, 42.50.Hz

\section{INTRODUCTION}

Spatiotemporal patterns in the transverse direction of an optical field have now been widely studied theoretically and experimentally [1]. Studies of optical pattern formation share a number of aspects and techniques with general investigations of pattern formation in other physical systems [2], but they also have specific features such as the role of light diffraction and the vectorial degree of freedom associated with the polarization of the light electric field amplitude. A prototype simple model that has been very useful for the understanding of pattern formation in nonlinear optical cavities is a mean field model describing a Kerr medium in a cavity with flat mirrors and driven by a coherent plane-wave field $[3,4]$. This model was extended to take into account the polarization degrees of freedom in Refs. [5-7]. Some of the basic polarization mechanisms of pattern formation in alkali vapors or other nonlinear materials can be understood in terms of this simple model despite the fact that the model is too simple to give a complete description of alkali vapors. Furthermore, the relative simplicity of the model in Ref. [5] makes it worthwhile to study it in depth as a general prototype model for the basic understanding of vectorial patterns. A first study was undertaken in Ref. [5] for the case of linearly polarized driving field and the positive cavity detuning. A more detailed study in which the case of elliptically polarized driving field is also considered has been presented in Ref. [7].

Here we study vectorial pattern formation in a Kerr medium close to a two-photon resonance placed inside a ring

\footnotetext{
*Electronic address: http://www.imedea.uib.es/PhysDept/

${ }^{\dagger}$ Mailing address: Center for Nonlinear Phenomena and Complex Systems, Université Libre de Bruxelles, Campus Plaine, Blv. du Triomphe B.P 231, 1050 Bruxelles, Belgium.
}

cavity. Two-photon processes have been studied in propagation in nonlinear Kerr media without any cavity as a way to realize optical phase conjugation mirrors. An ideal phase conjugate mirror should generate an output field such that the amplitude of the field, its propagation vector, and its polarization unit vector are the complex conjugates of the corresponding magnitudes in the input field. The first two properties can be achieved easily using, for example, four-wave mixing processes [8]. Usually the third property can be obtained only when the pump waves are circularly polarized and counterrotating. In order to find a system that verifies the three properties (vector phase conjugation) for an arbitrarily polarized input field it is required to make use of the special tensor properties of two-photon atomic transitions in degenerate four-wave mixing processes [9,10]. More precisely, vector phase conjugation can be achieved if the two levels coupled by the two-photon transition have equal angular momenta $J$ with $J=0$ or $J=1 / 2$. In this situation the $\chi_{1122}$ component of the susceptibility tensor vanishes. Intuitively, as $\Delta J=0$, the atom does not change its angular momentum either by absorbing two pump photons or by emitting a probe and a conjugate photon so that the conjugate photon must be emitted with angular momentum equal and opposite to that of the probe photon. A detailed calculation [9] shows that indeed this is true for the cases indicated before. We should stress that the polarization properties of two-photon-resonant degenerate four-wave mixing processes are different from those of most other degenerate four-wave mixing processes. In the two-photon case the underlying physical mechanism is scattering of the probe field from a spatially uniform temporally varying coherence induced by the two pump waves whereas in the other cases it is scattering from a spatially varying refractive-index distribution induced by the interference between the pump and the probe beams. Experimentally, vector phase conjugation was first observed using the $3 S_{1 / 2} \rightarrow 6 S_{1 / 2}$ two-photon transition in sodium vapor [11]. 
Here, instead of a cavityless Kerr medium, we are considering a Kerr medium inside a ring cavity. The same kind of Kerr medium is used, namely, the medium has a susceptibility tensor such that $\chi_{1122}=0$, close to a two-photon resonance. We will show that in this situation, when the cavity is illuminated with linearly polarized input and with negative detuning a new interesting situation appears: a codimension two bifurcation in which two stationary Turing-like instabilities occur simultaneously. The first instability, if the other were not present, would originate a hexagonal pattern that is polarized linearly in the same direction as the input field. The second instability is of pure vectorial origin and if the first instability were not present it would give rise to an elliptically polarized stripe pattern. Here we study the interplay between the two instabilities. The codimension two bifurcation appears here in a natural way associated to the twophoton-resonant four-wave mixing nonlinearities rather than as the result of the finetuning of two system parameters as is usually the case. The intensity of the pump field is the single control parameter to be tuned to change the distance to the instabilities. Furthermore, the system still has another easily accessible control parameter, the detuning, which allows the system to form different patterns while remaining at the same distance from the codimension two instability threshold. In particular, we show how the detuning can be used as a tuning parameter to select the pattern.

The outline of this paper is as follows. In Sec. II we describe the model we are considering, its spatially homogeneous solution, and the stability analysis of this solution. In Sec. III, using a weakly nonlinear analysis, we derive the evolution equation for the patterns arising from the interacting instabilities. From these equations, the selected patterns for different values of the detuning are analyzed in Sec. IV. In Sec. V we describe the results from numerical simulations of the model and finally in Sec. VI we give some concluding remarks.

\section{DESCRIPTION OF THE MODEL, REFERENCE STEADY STATES, AND STABILITY ANALYSIS}

The system we consider is a ring cavity filled with an isotropic Kerr medium. The cavity is driven by an external $\hat{x}$ polarized input field. The situation in which the polarization degree of freedom of the electromagnetic field is frozen was first considered by Lugiato and Lefever [3,4]. Geddes et al. [5] generalized the model of Ref. [3] to allow for the vector nature of the field. Their description of this system is given by a pair of coupled equations for the evolution of the two circularly polarized components of the field envelope $E_{+}$and $E_{-}$, defined by

$$
E_{ \pm}=\frac{1}{\sqrt{2}}\left(E_{x} \pm i E_{y}\right)
$$

For an isotropic medium, the equations are

$$
\begin{aligned}
\frac{\partial E_{ \pm}}{\partial t}= & -(1+i \eta \theta) E_{ \pm}+i a \nabla^{2} E_{ \pm}+E_{0}+i \eta\left[A\left|E_{ \pm}\right|^{2}\right. \\
& \left.+(A+B)\left|E_{\mp}\right|^{2}\right] E_{ \pm},
\end{aligned}
$$

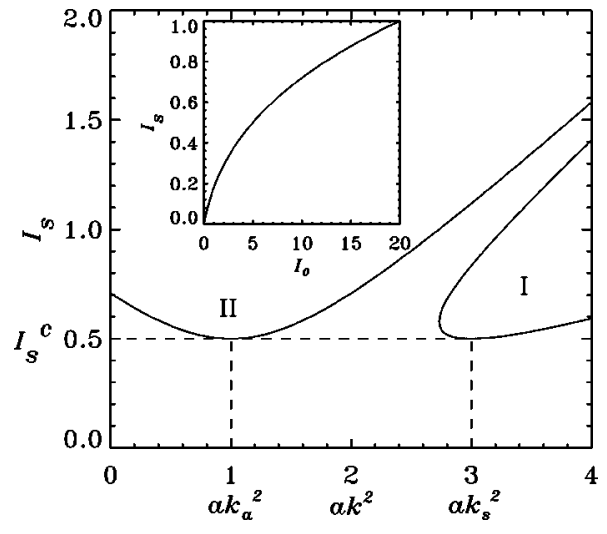

FIG. 1. Marginal stability curves for a linearly polarized input field corresponding to the symmetric solution. In the inset the symmetric steady state homogeneous solution is shown, as a function of the input field intensity, for linearly polarized light. Value of the detuning $\theta=-1$. The parameters used in Eq. (1) in order to get the codimension two situation are $\eta=1, a=1, A=0$, and $B=2$. The quantities plotted in all the figures are dimensionless.

where $E_{0}$ represents the components of the input field (the right and left circularly polarized components are equal since we consider $\hat{x}$ linearly polarized input), $\eta=+1(-1)$ indicates self-focusing (self-defocusing), $\theta$ is the cavity detuning, $a$ represents the strength of diffraction and $\nabla^{2}$ is the transverse Laplacian. The parameters $A$ and $B$ are related to the nonlinear susceptibility tensor components in the following way. $A=6 \chi_{1122}$ and $B=6 \chi_{1221}$ [8]. Also, for an isotropic medium we have $A+B / 2=1$. As discussed in the introduction, here we are considering two-photon transitions between levels with equal angular momenta $J$ where $J=0$ or $J=1 / 2$, so that $\chi_{1122}=0(A=0$ and $B=2)$. Also as we consider the self-focusing situation, in what follows we take $\eta=+1$. The intensity of the input field is $I_{0}=2\left|E_{0}\right|^{2}$.

The steady state homogeneous solutions of Eq. (1) are reference states from which transverse patterns emerge as they become unstable. There is a symmetric $\left(E_{s+}=E_{s-}\right.$ $\left.=E_{s}\right)$ and two asymmetric $\left(E_{s+} \neq E_{s-}\right)$ homogeneous solutions [7]. The symmetric solution corresponds to linearly polarized output light, while the asymmetric solutions to elliptically polarized output. Increasing the input field, the asymmetric solutions appear only for values of $I_{0}$ larger than the instability threshold for pattern formation [7], so here we will only consider the symmetric solution [12],

$$
I_{0} / 2=I_{s}\left[1+\left(2 I_{s}-\theta\right)^{2}\right]
$$

which gives an implicit formula for $I_{s}=\left|E_{s}\right|^{2}$. As is well known, Eq. (2) implies bistability for $\theta>\sqrt{3}$. However, here we will always consider negative detunings that are far away from the bistable regime. An example of the symmetric solution for linearly polarized input is given in the inset of Fig. 1.

Basic features of the stability of the steady state homogeneous symmetric solution can be analyzed by considering the evolution equations for perturbations $\psi_{ \pm}$defined by 


$$
E_{ \pm}=E_{s}\left(1+\psi_{ \pm}\right)
$$

From Eqs. (1) and (3) we find

$$
\begin{aligned}
\partial_{t} \psi_{ \pm}= & -\left[1+i\left(\theta-2 I_{s}\right)-i a \nabla^{2}\right] \psi_{ \pm} \\
& +i I_{s} 2\left(\psi_{\mp}+\psi_{\mp}^{*}+\left|\psi_{\mp}\right|^{2}\right)\left(1+\psi_{ \pm}\right) .
\end{aligned}
$$

It is convenient to make a change of variables to the following basis [5]:

$$
\begin{gathered}
\Sigma=\left(\sigma_{1}, \sigma_{2}, \sigma_{3}, \sigma_{4}\right)^{T}=\left[\operatorname{Re}\left(\psi_{+}+\psi_{-}\right),\right. \\
\left.\operatorname{Im}\left(\psi_{+}+\psi_{-}\right), \quad \operatorname{Re}\left(\psi_{+}-\psi_{-}\right), \quad \operatorname{Im}\left(\psi_{+}-\psi_{-}\right)\right]^{T},
\end{gathered}
$$

where $T$ stands for transpose. In this basis, which emphasizes the role of symmetric $\left(\psi_{+}=\psi_{-}\right)$and antisymmetric $\left(\psi_{+}=\right.$ $-\psi_{-}$) modes, Eq. (4) may be written as

$$
\partial_{t} \Sigma=L \Sigma+N_{2}(\Sigma \mid \Sigma)+N_{3}(\Sigma|\Sigma| \Sigma)
$$

where the linear matrix $L$ is a matrix with $2 \times 2$ blocks in which the symmetric $\left(\sigma_{1}, \sigma_{2}\right)$ and antisymmetric $\left(\sigma_{3}, \sigma_{4}\right)$ modes are decoupled,

$$
L=\left(\begin{array}{cc}
L_{1} & 0 \\
0 & L_{2}
\end{array}\right)
$$

As a consequence, the linear instabilities lead to the growth of either a symmetric or an antisymmetric mode. In Fourier space we have

$$
L_{1}=\left(\begin{array}{cc}
-1 & \left(\theta-2 I_{s}+a k^{2}\right) \\
-\left(\theta-6 I_{s}+a k^{2}\right) & -1
\end{array}\right)
$$

and

$$
L_{2}=\left(\begin{array}{cc}
-1 & \left(\theta-2 I_{s}+a k^{2}\right) \\
-\left(\theta+2 I_{s}+a k^{2}\right) & -1
\end{array}\right) \text {, }
$$

where $k \equiv|\vec{k}|$.

Instability occurs if at least one of the eigenvalues $\lambda$ of $L_{1}$ and $L_{2}$ has a positive real part. In Fourier space, these eigenvalues are solutions of the characteristic equations:

$$
\begin{aligned}
& \left(\lambda_{1}+1\right)^{2}+\left(\theta+a k^{2}-6 I_{s}\right)\left(\theta+a k^{2}-2 I_{s}\right)=0, \\
& \left(\lambda_{2}+1\right)^{2}+\left(\theta+a k^{2}+2 I_{s}\right)\left(\theta+a k^{2}-2 I_{s}\right)=0 .
\end{aligned}
$$

For $\theta<0$, we have

$$
\begin{aligned}
& \lambda_{1 \pm}=-1 \pm \sqrt{4 I_{s}^{2}-a^{2}\left(k^{2}-k_{s}^{2}\right)^{2}}, \\
& \lambda_{2 \pm}=-1 \pm \sqrt{4 I_{s}^{2}-a^{2}\left(k^{2}-k_{a}^{2}\right)^{2}},
\end{aligned}
$$

where $a k_{s}^{2}=4 I_{s}+|\theta|$ and $a k_{a}^{2}=|\theta|$. For both eigenvalues, the instability occurs at $I_{s}=1 / 2$.

The nonlinearities in Eq. (6) include quadratic $N_{2}(\Sigma \mid \Sigma)$ and cubic terms $N_{3}(\Sigma|\Sigma| \Sigma)$ :

$$
N_{2}(\Sigma \mid \Sigma)=I_{s}\left(\begin{array}{c}
2 \sigma_{3} \sigma_{4}-2 \sigma_{1} \sigma_{2} \\
3 \sigma_{1} \sigma_{1}+\sigma_{2} \sigma_{2}-\sigma_{3} \sigma_{3}+\sigma_{4} \sigma_{4} \\
2 \sigma_{2} \sigma_{3}-2 \sigma_{1} \sigma_{4} \\
-2 \sigma_{1} \sigma_{3}-2 \sigma_{2} \sigma_{4}
\end{array}\right)
$$

and

$N_{3}(\Sigma|\Sigma| \Sigma)$

$$
=\frac{I_{s}}{2}\left(\begin{array}{c}
2 \sigma_{1} \sigma_{3} \sigma_{4}-\sigma_{2}\left(\sigma_{1} \sigma_{1}+\sigma_{2} \sigma_{2}-\sigma_{3} \sigma_{3}+\sigma_{4} \sigma_{4}\right) \\
\sigma_{1}\left(\sigma_{1} \sigma_{1}+\sigma_{2} \sigma_{2}+\sigma_{3} \sigma_{3}-\sigma_{4} \sigma_{4}\right)-2 \sigma_{2} \sigma_{3} \sigma_{4} \\
2 \sigma_{1} \sigma_{2} \sigma_{3}-\sigma_{4}\left(\sigma_{1} \sigma_{1}-\sigma_{2} \sigma_{2}+\sigma_{3} \sigma_{3}+\sigma_{4} \sigma_{4}\right) \\
\sigma_{3}\left(-\sigma_{1} \sigma_{1}+\sigma_{2} \sigma_{2}+\sigma_{3} \sigma_{3}+\sigma_{4} \sigma_{4}\right)-2 \sigma_{1} \sigma_{2} \sigma_{4}
\end{array}\right) .
$$

The structure of these terms also gives some general information on the nature of the instabilities. In particular, if the quadratic nonlinearity $N_{2}(\Sigma \mid \Sigma)$ does not vanish, one expects the formation of a hexagonal pattern instead of stripes. As explained in Ref. [7], when the symmetric mode becomes unstable an hexagonal pattern is expected whereas when the antisymmetric mode becomes unstable there are no relevant quadratic couplings so a stripe pattern is formed.

In Fig. 1 we plot marginal stability curves for $\theta=-1$ as a function of $a k^{2}$. The shape of the marginal stability curves is, in fact, the same for any value of the detuning $\theta$. This is because the eigenvalues $\lambda_{i}$ given by Eq. (11) depend on $a k^{2}-|\theta|$, so a change in the value of $\theta$ is equivalent to a displacement of the origin of $a k^{2}$ by the same amount. The origin moves to the right if the detuning $\theta$ is increased.

The instability region I comes from the eigenvalue $\lambda_{1}$ so the critical modes are symmetric and of zero frequency. A subcritical hexagonal pattern is expected via a transcritical bifurcation. If this were the only instability, it would correspond to the case discussed in Ref. [4], in which the polarization degree of freedom is not taken into account. This instability leads to an $\hat{x}$-polarized pattern while the $\hat{y}$-polarized component of the field continues to be zero.

The instability region II comes from the eigenvalue $\lambda_{2+}$ so the critical modes are antisymmetric and of zero frequency. A stripe pattern is expected [5]. Given the antisymmetric nature of the unstable mode, the $\hat{x}$-polarized component of the field is stable and remains almost homogeneous, while the stripe pattern appears in the $\hat{y}$-polarized component, which has zero value below the instability. Overall, the electric field displays an elliptically polarized spatial structure. We remark that such an instability is of pure vectorial nature with no analog when the polarization degree of freedom is frozen.

In the case considered here, $\theta \leqslant 0$, starting from the linearly polarized homogeneous solution, as the input field is increased, the system crosses the two instability thresholds simultaneously. This is a codimension two bifurcation involving two sets of stationary modes. The critical modes associated to region I are symmetric and have a critical wave number $k_{s}$, while the critical modes associated to region II 
are antisymmetric and have a critical wave number $k_{a}$. The ratio $k_{a} / k_{s}$ can be changed easily varying the value of $\theta$.

\section{WEAKLY NONLINEAR ANALYSIS FOR INTERACTING TURING INSTABILITIES}

The eigenmodes of the linear evolution matrix $L$ are, in Fourier space $\quad\left[\hat{S}_{+}(\vec{k}), 0,0,0\right]^{T}, \quad\left[0, \hat{S}_{-}(\vec{k}), 0,0\right]^{T}$, $\left[0,0, \hat{A}_{+}(\vec{k}), 0\right]^{T}$, and $\left[0,0,0, \hat{A}_{-}(\vec{k})\right]^{T}$, with

$$
\begin{aligned}
& \hat{S}_{ \pm}(\vec{k})=\hat{\sigma}_{1}(\vec{k}) \pm \beta_{s}(k) \hat{\sigma}_{2}(\vec{k}), \\
& \hat{A}_{ \pm}(\vec{k})=\hat{\sigma}_{3}(\vec{k}) \mp \beta_{a}(k) \hat{\sigma}_{4}(\vec{k}),
\end{aligned}
$$

where $\hat{U}(\vec{k})=\mathcal{F}[U](\vec{k})$ denotes the Fourier transform of $U(\vec{r})$. Furthermore

$$
\beta_{j}(k)=\frac{2 I_{s}+a\left(k^{2}-k_{j}^{2}\right)}{\sqrt{4 I_{s}^{2}-a^{2}\left(k^{2}-k_{j}^{2}\right)^{2}}},
$$

where index $j$ stands for $s$ or $a$ and $\beta_{j}\left(k_{j}\right)=1$. The critical modes correspond to the eigenvalues $\lambda_{1+}\left(k_{s}\right)$ and $\lambda_{2+}\left(k_{a}\right)$. Note also that

$$
\begin{array}{cc}
\hat{\sigma}_{1}=\frac{\hat{S}_{+}+\hat{S}_{-}}{2}, & \hat{\sigma}_{2}=\frac{\hat{S}_{+}-\hat{S}_{-}}{2 \beta_{s}}, \\
\hat{\sigma}_{3}=\frac{\hat{A}_{+}+\hat{A}_{-}}{2}, & \hat{\sigma}_{4}=-\frac{\hat{A}_{+}-\hat{A}_{-}}{2 \beta_{a}} .
\end{array}
$$

After diagonalization of the linear evolution matrix, the dynamics (6) may be rewritten, in Fourier space, as

$$
\partial_{t} \hat{S}(\vec{k})=\hat{\Lambda}(\vec{k}) \hat{S}(\vec{k})+\left.\hat{N}_{2}(\hat{S} \mid \hat{S})\right|_{\vec{k}}+\left.\hat{N}_{3}(\hat{S}|\hat{S}| \hat{S})\right|_{\vec{k}},
$$

where $\hat{S}=\left(\hat{S}_{+}, \hat{S}_{-}, \hat{A}_{+}, \hat{A}_{-}\right)^{T}$ and $\hat{\Lambda}$ is a diagonal matrix with diagonal elements $\left\{\lambda_{1+}, \lambda_{1-}, \lambda_{2+}, \lambda_{2-}\right\} . \hat{N}_{2} \mid \vec{k}$ and $\left.\hat{N}_{3}\right|_{k}$ are the Fourier transforms of the nonlinear terms of Eq. (6), where the $\sigma_{i}$ have been replaced by the corresponding linear combinations of $S_{+}, S_{-}, A_{+}$, and $A_{-}$. Slightly above threshold, this dynamics may be reduced to the dynamics of the critical modes amplitudes only, through the adiabatic elimination of the stable noncritical modes. This procedure is now standard [14], and we will only sketch here the main steps of its application to model (6), and derive evolution equations for the critical modes, up to cubic nonlinearities.

Let us write the amplitudes of the critical modes as $S(\vec{k}) \equiv \hat{S}_{+}(\vec{k}) \delta\left(|\vec{k}|-k_{s}\right)$ and $A(\vec{k}) \equiv \hat{A}_{+}(\vec{k}) \delta\left(|\vec{k}|-k_{a}\right)$. Their dynamics writes

$$
\begin{aligned}
& \dot{S}(\vec{k})=\left(2 I_{s}-1\right) S(\vec{k})+\left(\left.\hat{N}_{2}\right|_{\vec{k}, k=k_{s}}\right)_{S}+\left(\left.\hat{N}_{3}\right|_{\vec{k}, k=k_{s}}\right)_{S}, \\
& \dot{A}(\vec{k})=\left(2 I_{s}-1\right) A(\vec{k})+\left(\left.\hat{N}_{2}\right|_{\vec{k}, k=k_{a}}\right)_{A}+\left(\left.\hat{N}_{3}\right|_{\vec{k}, k=k_{a}}\right)_{A} .
\end{aligned}
$$

The terms in the quadratic nonlinearities $\hat{N}_{2}$ are convolutions of products of critical and noncritical modes. The terms involving noncritical modes only $\left(\hat{S}_{-}^{2}, \hat{A}_{-}^{2}, \hat{S}_{-} \hat{A}_{-}\right)$may be ne- glected, since they contribute, through the adiabatic elimination process, to nonlinearities of quartic or higher order. Furthermore, the structure of the cubic nonlinearities of Eq. (6) is such that the pure critical mode contribution to $\hat{N}_{3}$ vanishes. Hence $\hat{N}_{3}$ will be neglected. We are thus dealing here with a nongeneric case, where cubic nonlinearities are generated solely by the adiabatic elimination of stable modes from quadratic terms. The nonlinearities $\left(\left.\hat{N}_{2}\right|_{\vec{k}, k=k_{s}}\right)_{S}$ and $\left(\left.\hat{N}_{2}\right|_{\vec{k}, k=k_{s}}\right)_{A}$ can be written at the lowest order in noncritical modes as

$$
\begin{aligned}
\left(\hat{N}_{2} \mid \vec{k}_{k=k_{s}}\right)_{S} \simeq & \frac{I_{s}}{2} \int d \vec{k}^{\prime}\left[S\left(\vec{k}-\vec{k}^{\prime}\right) S\left(\vec{k}^{\prime}\right)-A\left(\vec{k}-\vec{k}^{\prime}\right) A\left(\vec{k}^{\prime}\right)\right. \\
& +2 S\left(\vec{k}-\vec{k}^{\prime}\right) \hat{S}_{-}\left(\vec{k}^{\prime}\right) \delta\left(\left|\vec{k}^{\prime}\right|-k_{s}\right) \\
& \left.-2 A\left(\vec{k}-\vec{k}^{\prime}\right) \hat{A}_{-}\left(\vec{k}^{\prime}\right) \delta\left(\left|\vec{k}^{\prime}\right|-k_{a}\right)\right] \\
& +I_{s}\left\{S(\vec{k})\left[\hat{S}_{+}(0)+\hat{S}_{-}(0)\right]+S(-\vec{k})\right. \\
\times & \left.\left.\times \hat{S}_{+}(2 \vec{k})+\hat{S}_{-}(2 \vec{k})\right]\right\} \\
\left(\hat{N}_{2} \mid \vec{k}, k=k_{a}\right)_{A} \simeq & I_{s} \int d \vec{k}^{\prime}\left[S\left(\vec{k}-\vec{k}^{\prime}\right) A\left(\vec{k}^{\prime}\right)\right. \\
& +S\left(\vec{k}-\vec{k}^{\prime}\right) \hat{A}_{-}\left(\vec{k}^{\prime}\right) \delta\left(\left|\vec{k}^{\prime}\right|-k_{a}\right) \\
& \left.+A\left(\vec{k}-\vec{k}^{\prime}\right) \hat{S}_{-}\left(\vec{k}^{\prime}\right) \delta\left(\left|\vec{k}^{\prime}\right|-k_{s}\right)\right] \\
& +I_{s} A(\vec{k})\left[\hat{S}_{+}(0)+\hat{S}_{-}(0)\right] .
\end{aligned}
$$

The noncritical modes $\hat{S}_{ \pm}(0), \hat{S}_{ \pm}\left(2 \vec{k}_{s}\right), \quad \hat{S}_{-}\left(\vec{k}_{s}\right)$, and $\hat{A}_{-}\left(\vec{k}_{a}\right)$ present in the Eqs. (19) may be expressed as an expansion in powers of critical ones using the adiabatic elimination procedure. One has at the leading order

$$
\begin{gathered}
\hat{S}_{ \pm}(0)=\frac{I_{s}}{2 \lambda_{1, \pm}(0)} \int d \vec{k}\left\{A(\vec{k}) A(-\vec{k})+\left[1 \mp 2 \beta_{s}(0)\right]\right. \\
\times S(\vec{k}) S(-\vec{k})\}, \\
\hat{S}_{ \pm}\left(2 \vec{k}_{s}\right)=\frac{I_{s}}{2 \lambda_{1, \pm}\left(2 k_{s}\right)}\left[1 \mp 2 \beta_{s}\left(2 k_{s}\right)\right] S\left(\vec{k}_{s}\right) S\left(\vec{k}_{s}\right), \\
\hat{S}_{-}\left(\vec{k}_{s}\right)=\frac{I_{s}}{2 \lambda_{1,-}\left(k_{s}\right)} \int d \vec{k}^{\prime}\left[3 S\left(\vec{k}_{s}-\vec{k}^{\prime}\right) S\left(\vec{k}^{\prime}\right)\right. \\
\left.\quad+A\left(\vec{k}_{s}-\vec{k}^{\prime}\right) A\left(\vec{k}^{\prime}\right)\right], \\
\hat{A}_{-}\left(\vec{k}_{a}\right)=-\frac{I_{s}}{\lambda_{2,-}\left(k_{a}\right)} \int d \vec{k}^{\prime} S\left(\vec{k}_{a}-\vec{k}^{\prime}\right) A\left(\vec{k}^{\prime}\right) .
\end{gathered}
$$

The substitution of Eqs. (20) in Eqs. (19) leads to the following asymptotic dynamics for the critical modes, valid close to the instability threshold 


$$
\begin{aligned}
& \dot{S}(\vec{k})=\left(2 I_{s}-1\right) S(\vec{k})+\int d \vec{k}^{\prime}\left[v_{0} S\left(\vec{k}-\vec{k}^{\prime}\right) S\left(\vec{k}^{\prime}\right)\right. \\
&\left.+v_{1} A\left(\vec{k}-\vec{k}^{\prime}\right) A\left(\vec{k}^{\prime}\right)\right]-\int d \vec{k}^{\prime} \int d \vec{k}^{\prime \prime} u\left(\vec{k}, \vec{k}^{\prime}, \vec{k}^{\prime \prime}\right) \\
& \times S\left(\vec{k}-\vec{k}^{\prime}\right) S\left(\vec{k}^{\prime}-\vec{k}^{\prime \prime}\right) S\left(\vec{k}^{\prime \prime}\right) \\
&-\int d \vec{k}^{\prime} \int d \vec{k}^{\prime \prime} w\left(\vec{k}, \vec{k}^{\prime}, \vec{k}^{\prime \prime}\right) \\
& \times S\left(\vec{k}-\vec{k}^{\prime}\right) A\left(\vec{k}^{\prime}-\vec{k}^{\prime \prime}\right) A\left(\vec{k}^{\prime \prime}\right), \\
& \dot{A}(\vec{k})=\left(2 I_{s}-1\right) A(\vec{k})+v_{2} \int d \vec{k}^{\prime} S\left(\vec{k}-\vec{k}^{\prime}\right) A\left(\vec{k}^{\prime}\right) \\
&- \int d \vec{k}^{\prime} \int d \vec{k}^{\prime \prime} \bar{w}\left(\vec{k}, \vec{k}^{\prime}, \vec{k}^{\prime \prime}\right) A\left(\vec{k}-\vec{k}^{\prime}\right) A\left(\vec{k}^{\prime}-\vec{k}^{\prime \prime}\right) A\left(\vec{k}^{\prime \prime}\right) \\
&- \int d \vec{k}^{\prime} \int d \vec{k}^{\prime \prime} \bar{u}\left(\vec{k}, \vec{k}^{\prime}, \vec{k}^{\prime \prime}\right) A\left(\vec{k}-\vec{k}^{\prime}\right) S\left(\vec{k}^{\prime}-\vec{k}^{\prime \prime}\right) S\left(\vec{k}^{\prime \prime}\right),
\end{aligned}
$$

where $v_{0}=-v_{1}=v_{2} / 2=I_{s} / 2$, and

$$
\begin{gathered}
u\left(\vec{k}, \vec{k}^{\prime}, \vec{k}^{\prime \prime}\right)=u_{1} \delta\left(\left|\vec{k}^{\prime}\right|-k_{s}\right)+u_{2} \delta\left(\vec{k}^{\prime}\right)+u_{3} \delta\left(\vec{k}^{\prime}\right) \delta\left(\vec{k}^{\prime \prime}-\vec{k}\right) \\
w\left(\vec{k}, \vec{k}^{\prime}, \vec{k}^{\prime \prime}\right)=w_{1} \delta\left(\left|\vec{k}^{\prime}\right|-k_{s}\right)+w_{2} \delta\left(\vec{k}^{\prime}\right) \\
+w_{3} \delta\left(\left|\vec{k}-\vec{k}^{\prime}-\vec{k}^{\prime \prime}\right|-k_{a}\right), \\
\bar{u}\left(\vec{k}, \vec{k}^{\prime}, \vec{k}^{\prime \prime}\right)=u_{1} \delta\left(\left|\vec{k}^{\prime}\right|-k_{s}\right)+u_{2} \delta\left(\vec{k}^{\prime}\right)-w_{3} \delta\left(\left|\vec{k}-\vec{k}^{\prime}\right|-k_{a}\right) \\
\bar{w}\left(\vec{k}, \vec{k}^{\prime}, \vec{k}^{\prime \prime}\right)=w_{1} \delta\left(\left|\vec{k}^{\prime}\right|-k_{s}\right)+w_{2} \delta\left(\vec{k}^{\prime}\right),
\end{gathered}
$$

with $u_{1}=3 I_{s}^{2} /\left(2+4 I_{s}\right), \quad u_{2}=I_{s}^{2}(3+2|\theta|) /(2+|\theta|)^{2}, \quad u_{3}=$ $-\left[I_{s}^{2} / 9(13+6|\theta|)\right] /\left[(2+|\theta|)^{2}\right], w_{1}=w_{3}=I_{s}^{2} /\left(2+4 I_{s}\right)$, and $w_{2}=I_{s}^{2} /(2+|\theta|)^{2}$. It has to be noted that the $u$ terms come from quadratic resonances between critical and noncritical symmetric modes, while the $w$ terms come from quadratic resonances between symmetric and antisymmetric modes. As in the case of isolated Turing-like instabilities, there is no quadratic resonance between critical antisymmetric modes only. As a result, pattern formation is expected to strongly depend on the existence of quadratic resonances between symmetric and antisymmetric modes. Hence, for the sake of simplicity, we will consider separately the case with quadratic resonance between symmetric modes only, and the case with quadratic couplings between antisymmetric and symmetric modes, which is more intricate. In the latter case, quadratic couplings are such that $\vec{k}_{1}=\vec{k}_{2}+\vec{k}_{3}$, with $\left|\vec{k}_{1}\right|=k_{s}$ and $\left|\vec{k}_{2,3}\right|=k_{a}$. Since $\cos \phi=k_{s} / 2 k_{a}$, where $\phi$ is the angle between symmetric and antisymmetric vectors, quadratic resonance arise only for $|\theta|>4 I_{s} / 3$.

\section{PATTERN SELECTION AND STABILITY}

In this section we study the various patterns that may appear as asymptotic solutions of Eq. (21), and their stability. Each of these patterns is built on an arbitrary number of critical modes pairs, and it is the nature of their nonlinear couplings that determines their stability. In the following, we label the modes contributing to the formation of a pattern $S\left(\vec{k}_{i}\right)=S_{i}, A\left(\vec{k}_{i}\right)=A_{i}$. We also use the notation $\bar{S}_{i}$ for the complex conjugate of $S_{i}$.

\section{A. $k_{a}<k_{s} / 2$ or $|\theta|<4 I_{s} / 3$}

In this case, there is no quadratic coupling between symmetric and antisymmetric modes, and there is no contribution coming from the terms with the coefficients $v_{1}, v_{2}, w_{1}$, and $w_{3}$. Let us then consider separately amplitude equations for each type of modes.

\section{Antisymmetric modes}

For patterns built on antisymmetric modes only, the amplitude equations for an arbitrary number $m$ of pairs of modes are

$$
\dot{A}_{i}=\left(2 I_{s}-1\right) A_{i}-2 w_{2} A_{i} \sum_{j=1}^{m}\left|A_{j}\right|^{2} .
$$

Hence, a pattern built on $m$ pairs of wave vectors is marginally stable versus a $m+1$ pair of wave vectors. So, at this level of analysis, any pattern with an arbitrary number of wave vectors is possible, including patterns of the form $A J_{0}\left(k_{a} r\right)$, where $J_{0}$ is zeroth order Bessel function and $A$ $=\sqrt{\left(2 I_{s}-1\right) / 2 w_{2}}$.

The linear growth rate of the evolution of symmetric modes in the presence of such patterns is zero, so antisymmetric patterns are marginally stable versus symmetric mode patterns. It would be necessary to go to higher orders in the amplitude equations to complete the pattern selection analysis in this case.

\section{Symmetric modes}

On the other hand, for patterns built on symmetric modes only, the amplitude equations for a triplet of such modes are:

$$
\begin{aligned}
\dot{S}_{i}= & \left(2 I_{s}-1\right) S_{i}+2 v_{0} \bar{S}_{i+1} \bar{S}_{i-1}-\left(2 u_{2}+u_{3}\right)\left|S_{i}\right|^{2} S_{i} \\
& -2\left(u_{1}+u_{2}\right) S_{i} \sum_{j \neq i}\left|S_{j}\right|^{2} .
\end{aligned}
$$

At the instability threshold $\left(I_{s}=\frac{1}{2}\right)$, hexagonal pattern appear via a subcritical bifurcation. Increasing the value of $I_{s}$, stripes may also become stable for $2 I_{s}-1 \geqslant[8(3$ $+2|\theta|)] /\left[9(2+|\theta|)^{2}\right]$. There is a small region of bistability of stripes and hexagons and for larger values of $I_{s}$ only the stripes remain stable.

Hexagonal patterns are stable versus antisymmetric modes for $I_{s} \leqslant(1 / 2)\left[1+(3+2|\theta|) / 4(2+|\theta|)^{2}\right]$. Hence, sufficiently close to threshold, one may expect hexagonal patterns. On increasing $I_{s}$, such patterns should become unstable. 


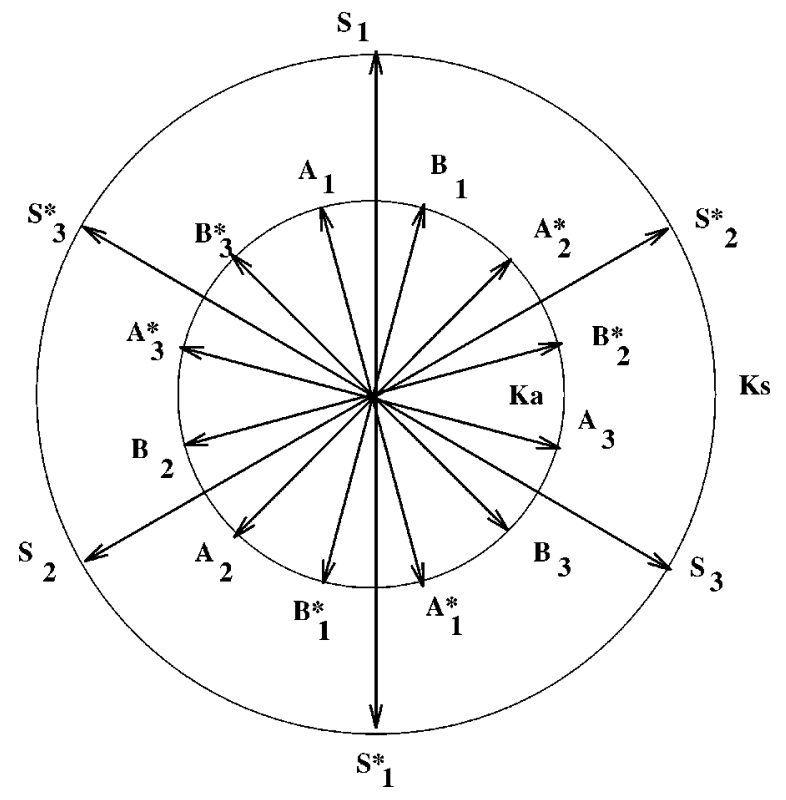

FIG. 2. Quadratically coupled symmetric and antisymmetric modes with wave numbers $k_{s}$ and $k_{a}$

\section{Mixed modes}

The amplitude equations for mixed structures formed by triplets of symmetric modes and an arbitrary number of antisymmetric modes are

$$
\begin{aligned}
\dot{S}_{i}= & \left(2 I_{s}-1\right) S_{i}+2 v_{0} S_{i+1} S_{i-1}-S_{i}\left[\left(2 u_{2}+u_{3}\right)\left|S_{i}\right|^{2}\right. \\
& \left.+2\left(u_{1}+u_{2}\right) \sum_{j=1}^{3}\left|S_{j}\right|^{2}+2 w_{2} \sum_{l=1}^{m}\left|A_{l}\right|^{2}\right], \\
\dot{A}_{k}= & \left(2 I_{s}-1\right) A_{k}-A_{k}\left[2 u_{2} \sum_{j=1}^{3}\left|S_{j}\right|^{2}+2 w_{2} \sum_{l=1}^{m}\left|A_{l}\right|^{2}\right] .
\end{aligned}
$$

However, these equations do not admit nontrivial steady states. As a result, in these conditions, hexagonal or striped patterns of symmetric modes, and patterns built on an arbitrary number of antisymmetric modes may be simultaneously stable.

\section{B. $k_{s} / 2<k_{a}<k_{s}$ or $|\theta|>4 I_{s} / 3$}

In this case, quadratic resonances may occur between symmetric and antisymmetric modes, and one may now expect contributions coming from the $v_{1}, v_{2}, w_{1}$, and $w_{3}$ in the amplitude equations, which have to be modified accordingly. Let us then consider the different types of patterns that may arise in this case, and which are built on modes belonging to the following set of critical modes (up to an arbitrary phase angle) (cf. Fig. 2).

\section{Symmetric modes}

Striped and hexagonal symmetric mode patterns are now always unstable versus antisymmetric modes. This is due to the positive nonlinear renormalization induced by these modes in the evolution equation for their resonantly coupled antisymmetric modes (this renormalization is associated to the terms with $-w_{3}$ coefficients in $\bar{u}$ ).

\section{Antisymmetric modes}

As in Sec. IV A, a pattern built on $m$ arbitrary pairs of antisymmetric modes is marginally stable versus a pattern with $m+1$ pairs of wave vectors. Furthermore, since the contributions of the quadratically resonant symmetric and antisymmetric modes, in their respective amplitude equations have opposite signs $\left(v_{1}<0\right.$ and $\left.v_{2}>0\right)$, pure antisymmetric mode patterns are also unstable versus resonantly coupled symmetric and antisymmetric ones. Recall also that a pattern built on $m$ arbitrary pairs of antisymmetric modes is marginally stable versus nonresonant symmetric modes.

\section{Mixed modes}

As a result, when quadratic resonances between symmetric and antisymmetric modes are possible, pure steady patterns built on symmetric or antisymmetric modes only, are always unstable. We have thus to consider the possibility for the system to develop mixed mode patterns.

Let us consider the simplest case of mixed mode patterns built on one symmetric mode and two quadratically resonant antisymmetric modes (e.g., $S_{1}, A_{1}$, and $B_{1}$ in Fig. 2). Their uniform amplitude equations are

$$
\begin{aligned}
\dot{S}_{1}= & \left(2 I_{s}-1\right) S_{1}-I_{s} A_{1} B_{1}-S_{1}\left[\left(2 u_{2}+u_{3}\right)\left|S_{1}\right|^{2}\right. \\
& \left.+2 w_{2}\left(\left|A_{1}\right|^{2}+\left|B_{1}\right|^{2}\right)\right], \\
\dot{A}_{1}= & \left(2 I_{s}-1\right) A_{1}+I_{s} S_{1} \bar{B}_{1}-A_{1}\left[2\left(u_{2}-w_{3}\right)\left|S_{1}\right|^{2}\right. \\
& \left.+\left(2 w_{2}\left|A_{1}\right|^{2}+\left(2 w_{2}+w_{1}\right)\left|B_{1}\right|^{2}\right)\right], \\
\dot{B}_{1}= & \left(2 I_{s}-1\right) B_{1}+I_{s} S_{1} \bar{A}_{1}-B_{1}\left[2\left(u_{2}-w_{3}\right)\left|S_{1}\right|^{2}\right. \\
& \left.+\left(2 w_{2}\left|B_{1}\right|^{2}+\left(2 w_{2}+w_{1}\right)\left|A_{1}\right|^{2}\right)\right] .
\end{aligned}
$$

Because of the symmetry between $A_{1}$ and $B_{1}$ we look for solutions with the same amplitude for both antisymmetric modes. Defining amplitude and phase variables as $S_{1}$ $=R_{s} \exp i \phi, A_{1}=R_{a} \exp i \psi, B_{1}=R_{a} \exp i \bar{\psi}$, and $\Psi=\phi-\psi$ $-\bar{\psi}$, one has

$$
\begin{gathered}
\dot{R}_{s}=\left(2 I_{s}-1\right) R_{s}-I_{s} R_{a}^{2} \cos \Psi-R_{s}\left[\left(2 u_{2}+u_{3}\right) R_{s}^{2}+4 w_{2} R_{a}^{2}\right] \\
\dot{R}_{a}=\left(2 I_{s}-1\right) R_{a}+I_{s} R_{a} R_{s} \cos \Psi-R_{a}\left[2\left(u_{2}-w_{3}\right) R_{s}^{2}\right. \\
\left.+\left(4 w_{2}+2 w_{1}\right) R_{a}^{2}\right] \\
\dot{\Psi}=\frac{I_{s}}{R_{s}}\left[R_{a}^{2}-2 R_{s}^{2}\right] \sin \Psi
\end{gathered}
$$

A phase stable steady state corresponds thus to $\Psi=2 n \pi$ if $R_{a}^{2}<2 R_{s}^{2}$, and to $\Psi=(2 n+1) \pi$ if $R_{a}^{2}>2 R_{s}^{2}$. Combining the steady state conditions for $R_{s}$ and $R_{a}$, it can be seen that $\Psi=2 n \pi$ requires that $R_{a}^{2}>R_{s}^{2}$ and that $\Psi=(2 n+1) \pi$ requires that $R_{a}^{2}<R_{s}^{2}$. As a result, a stable steady state may only be obtained for $\Psi=2 n \pi$, with $R_{s}^{2}<R_{a}^{2}<2 R_{s}^{2}$. Furthermore, this condition is satisfied if the kinetic coefficients are 
such that $1 \leqslant|\theta|<2(\sqrt{3}-1) \simeq 1.46$, which is thus a necessary condition to be satisfied to obtain such mixed modes solutions. This condition corresponds to $0.57 k_{s}<k_{a}$ $<0.65 k_{s}$ or $0.64<\cos \phi<0.769$, with $I_{s} \simeq 0.5$.

A similar analysis may be performed for a pattern formed by an hexagonal planform of symmetric modes and their quadratically resonant antisymmetric ones (cf. Fig. 2). However, in this case, the fact that the quadratic couplings between symmetric and antisymmetric modes have opposite signs does not allow the stabilization of critical patterns.

\section{Summary of the analytical results}

The conclusion of the analysis presented in the two preceding subsections is as follows.

For $k_{a}<0.5 k_{s}(|\theta|<0.666)$, close to threshold, hexagonal symmetric patterns are stable. For slightly larger values of $I_{s}$ symmetric stripes and hexagons are bistable and finally only the stripes remain stable. Patterns built on an arbitrary number of antisymmetric modes are neutrally stable. As these results come from an expansion up to cubic nonlinearities we cannot conclude about the stability of the antisymmetric patterns. It would be necessary to go to higher order terms. Finally, there are no mixed stationary patterns.

For $0.5 k_{s}<k_{a}<0.57 k_{s}(0.666<|\theta|<0.963)$, pure symmetric and antisymmetric mode patterns are unstable. Also, no steady mixed mode patterns are found either. For that reason, one expects to find time dependent structures that involve both symmetric as well as antisymmetric modes.

For $0.57 k_{s}<k_{a}<0.65 k_{s}(0.963<|\theta|<1.46)$, one may expect steady patterns formed by the superposition of one symmetric mode and its quadratically resonant antisymmetric ones. We should notice that the range in which these steady mixed patterns do exist may be in fact smaller than 0.963 $<|\theta|<1.46$ because these boundary values have been obtained as a necessary condition for the stability of the global phase. This is not a suficient condition to guarantee the stability of the pattern, which can undergo other instabilities (despite the global phase being stable). As we will see in the following section, the approximate bounds obtained via numerical integration for this case are slightly different.

For $0.65 k_{s} \leqslant k_{a}(1.46<|\theta|)$, symmetric modes are unstable versus antisymmetric modes and no steady mixed mode solutions are found. Patterns composed of an arbitrary number of antisymmetric modes are neutrally stable, so it would be necessary to include higher order nonlinearities to conclude about their stability.

The present analysis provides the basic elements for the study of pattern formation when both symmetric and antisymmetric modes become simultaneously unstable. As it will be discussed below, it is partially confirmed by numerical analysis of the complete dynamical model. It could be improved, on the one hand, in determining the full stability range of mixed mode patterns, and, on the other hand, in resolving the issue of finding asymptotic states in cases where no steady critical patterns are found. In such cases, as suggested by numerical analysis, one should consider the possibility of asymptotic time-dependent or noncritical patterns. The latter case would require to incorporate in the
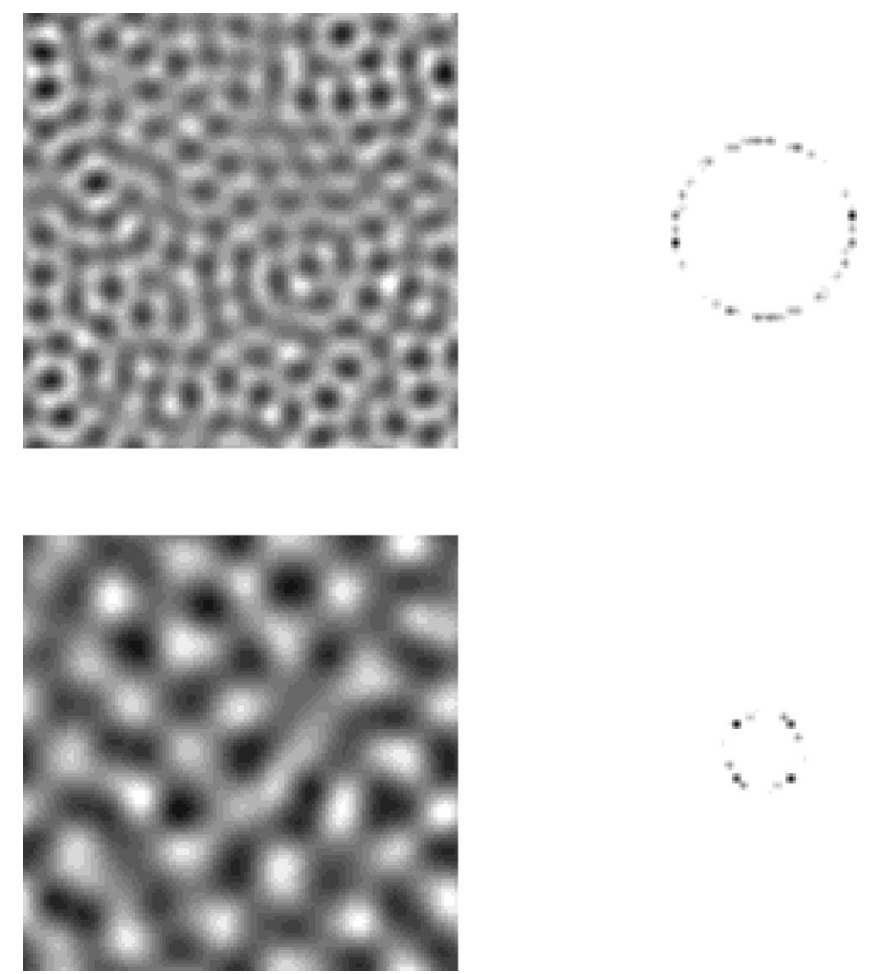

FIG. 3. Field configuration for $\theta=-0.5$ and $I_{s}=0.51 \quad\left(I_{0}\right.$ $=3.31$ ) after integrating Eq. (1) for a time $t=35000$. From left to right and from top to bottom: $\operatorname{Re}\left[E_{x}(\vec{r})\right]$ (near field $\hat{x}$-polarized component plotted with the grayscale: black $=0.51$, white $=0.60)$, $\left|E_{x}(\vec{k})\right|^{2}$ (far field $\hat{x}$ component), $\operatorname{Re}\left[E_{y}(\vec{r})\right]$ (near field $\hat{y}$-polarized component plotted with the grayscale: black $=-0.035$, white $=0.042)$, and $\left|E_{y}(\vec{k})\right|^{2}$ (far field $\hat{y}$ component).

dynamics harmonics or noncritical modes. We should notice that a peculiarity of the situation considered here is that there are no cubic nonlinear terms involving only critical modes. The cubic nonlinearities we have considered are generated solely by the adiabatic elimination of stable modes from the quadratic terms. However, for an input field that is slightly above threshold there will be a range of unstable modes around the critical one. The cubic nonlinearity $\hat{N}_{3}$ for these modes will be small but nonzero. As the cubic nonlinearities generated by the adiabatic elimination of stable modes from the quadratic terms are also small, it would be necessary to include in the analysis both kinds of cubic nonlinearities. This is beyond the scope of the present paper.

\section{NUMERICAL RESULTS}

We have performed several numerical integrations of Eq. (1) using a numerical scheme described in detail in Ref. [13]. The method is pseudospectral and second-order accurate in time, and is similar to the so-called two-step method. Periodic boundary conditions and lattices of size $128 \times 128$ were used.

For $|\theta|<0.666$ there is not a mixed mode pattern since quadratic resonance is not possible due to the fact that $k_{a}$ $<k_{s} / 2$. An example of this situation is shown in Fig. 3 for $\theta=-0.5$. According to the weakly nonlinear analysis, sta- 

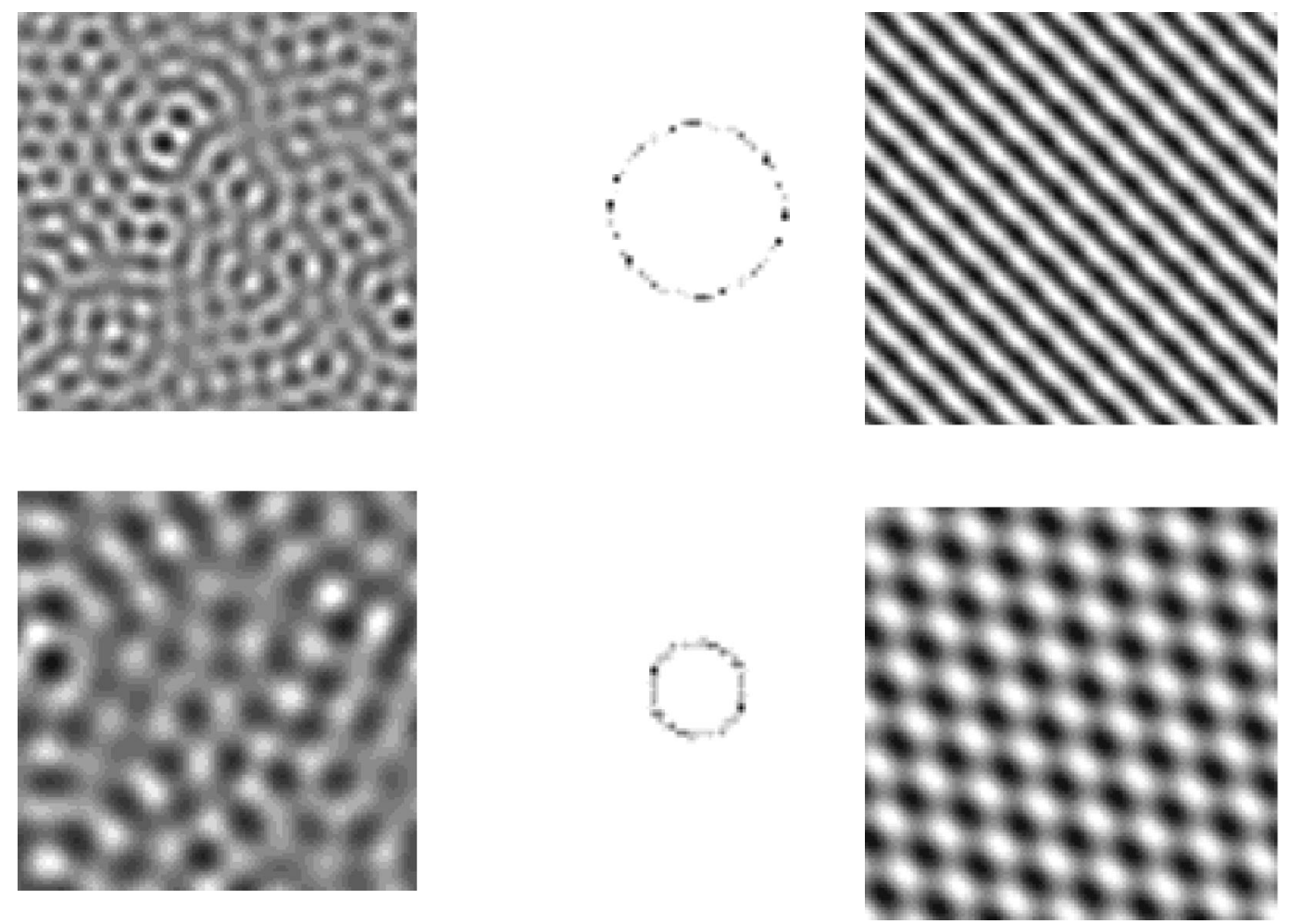

FIG. 4. Field configuration for $\theta=-0.83, I_{s}=0.51 \quad\left(I_{0}\right.$ $=4.41$ ), and an integration time of $t=65000$. From left to right and from top to bottom: $\operatorname{Re}\left[E_{x}(\vec{r})\right]$ (grayscale: black $=0.44$, white $=0.51$ ), $\left|E_{x}(\vec{k})\right|^{2}, \operatorname{Re}\left[E_{y}(\vec{r})\right]$ (grayscale: black $=-0.036$, white $=0.033)$, and $\left|E_{y}(\vec{k})\right|^{2}$.

tionary symmetric hexagons are stable close to threshold, however, we find numerically a disordered structure for both polarization components of the field $E_{x}$ and $E_{y}$. As $E_{x}$ $=\left(E_{+}+E_{-}\right) / \sqrt{2}$ and $E_{y}=\left(E_{+}-E_{-}\right) / \sqrt{2} i$, the structure observed in $E_{x}$ is formed by symmetric modes with wave vectors in the ring $|\vec{k}|=k_{s}$ whereas the structure in $E_{y}$ is composed of antisymmetric modes with wave vectors $|\vec{k}|=k_{a}$. The overall structure is built on an arbitrary number of symmetric and antisymmetric modes. The weakly nonlinear analysis predicted that there were no stationary mixed patterns and, in fact, the pattern shown in Fig. 3 from numerical integration is not static, it evolves dynamically in a slow time scale.

For $0.666<|\theta|<0.963$, although resonance is possible, no mixed mode pattern is numerically found, as predicted analytically. As in the previous case, a disordered structure is obtained and no steady pattern is reached even for long time integrations. Rings of radius $k_{s}$ and $k_{a}$ in the far fields of the linearly polarized components $\hat{x}$ and $\hat{y}$ respectively, show that there is not a selection process of critical wave vectors, all directions become unstable. In Fig. 4 we show the field configurations for $\theta=-0.83$.

The analysis of the preceding section showed that mixed mode patterns may be expected for $0.963<|\theta|<1.46$. In fact, for $\theta=-1$ a pattern formed by the superposition of a symmetric mode and the quadratically resonant antisymmetric ones is obtained, see Fig. 5. A roll pattern is seen in the $\hat{x}$

FIG. 5. Field configuration for $\theta=-1, I_{s}=0.51\left(I_{0}=5.06\right)$, and an integration time of $t=14000$. From left to right and from top to bottom: $\operatorname{Re}\left[E_{x}(\vec{r})\right] \quad$ (grayscale: black $=0.43$, white $=0.47$ ), $\left|E_{x}(\vec{k})\right|^{2}, \operatorname{Re}\left[E_{y}(\vec{r})\right]$ (grayscale: black $=-0.047$, white $=0.047$ ), and $\left|E_{y}(\vec{k})\right|^{2}$.

linearly polarized component with wave number $k_{s}$, while a rectangular pattern appears in the $\hat{y}$ component with wave number $k_{a}$. As for the range of existence of mixed mode patterns, we have found numerically the existence of these patterns also for $\theta=-1.05$, but not for $|\theta| \geqslant 1.1$.

For $\theta=-1.1$ we find a steady pattern in which the symmetric modes are damped and a stripe pattern with wave number $k_{a}$ appears in the $\hat{y}$-polarized component, while $x$-polarized component is almost homogeneous (in fact, a small amplitude stripe pattern of wave vector $2 k_{a}$ is observed in $E_{x}$, which comes from the coupling between $E_{x}$ and $E_{y}$ ). This pattern made out of antisymmetric modes is the same pattern that is formed when only the antisymmetric instability is present. Overall, the electric field displays an elliptically polarized spatial structure. Similar stationary patterns are found for larger absolute values of the detuning. In Fig. 6 we show the case with $\theta=-2$; this value was chosen in order to induce the formation of a square pattern, since the angle $\phi$ between symmetric and antisymmetric vectors is $\phi$ $=45^{\circ}$ in this case, but anyway the antisymmetric stripe pattern is formed as described.

\section{CONCLUDING REMARKS}

We have studied the spatial polarization structures in a mean field model for a Kerr medium close to a two-photon resonance and driven by a linearly $\hat{x}$-polarized field with 


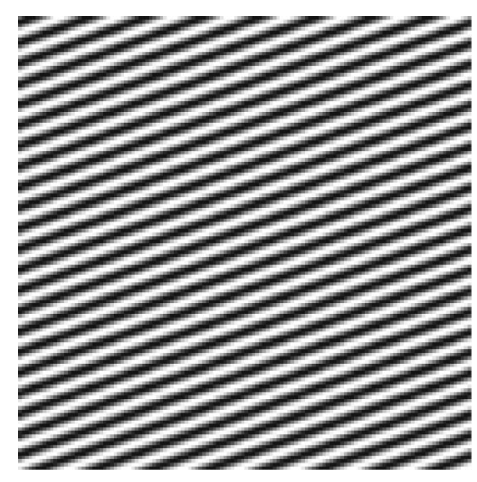

form of the nonlinearities associated to the two-photonresonant four-wave mixing process. As this codimension two bifurcation is not the result of the finetuning of two parameters, as it is usually the case, it should be much more simple to be observed. In fact, there is only one parameter, the pump, which has to be tuned. Furthermore, we still have another free parameter, the detuning, which allows the system to form different patterns without changing the distance to the codimension two instability threshold.

Near the instability threshold, we have obtained the evolution equation for the patterns arising from the interacting instabilities using a weakly nonlinear analysis. From these analysis and from the numerical integration of the model we have shown that we can have the following patterns: (a) dynamical structures involving an arbitrary number of symmetric and antisymmetric modes, (b) a steady state pattern formed by the superposition of a symmetric mode and the quadratically resonant antisymmetric ones, and (c) an antisymmetric stripe steady pattern in $E_{y}$, which is the same that would appear if only the antisymmetric instability were present.

As a final remark we notice that an interesting peculiarity of the situation considered here is that the selection among these patterns can be done in a very natural way, that is, changing the value of the detuning.

\section{ACKNOWLEDGMENTS}

FIG. 6. Field configuration for $\theta=-2, I_{s}=0.51\left(I_{0}=10.2\right)$, and an integration time of $t=10000$. From left to right and from top to bottom: $\operatorname{Re}\left[E_{x}(\vec{r})\right] \quad$ (grayscale: black $=0.32, \quad$ white $=0.34$ ), $\left|E_{x}(\vec{k})\right|^{2}, \operatorname{Re}\left[E_{y}(\vec{r})\right]$ (grayscale: black $=-0.15$, white $=0.15$ ), and $\left|E_{y}(\vec{k})\right|^{2}$.

negative detuning. In the self-focusing case the first pattern that is formed as the pump intensity is increased arises from the competition between two stationary instabilities that occur simultaneously. This codimension two bifurcation appears naturally in the system and it is a consequence of the

Helpful discussions with Dr. M. Santagiustina are acknowledged. This work is supported by the European Commission through the QSTRUCT TMR network (Project ERB FMRX-CT96-0077) and by MCyT (Spain) Projects PB941167, PB97-0141-C02-02, and BFM2000-1108. M. H. wants to acknowledge financial support from the FOMEC project 290, Departamento de Física FCEyN, Universidad Nacional de Mar del Plata, Argentina and from the National Council for Scientific and Technical Research (CONICET) of Argentina.

[1] Nonlinear Optical Structures, Patterns, Chaos, edited by L. A. Lugiato, special issue of Chaos, Solitons Fractals 4, 1251 (1994), and references therein.

[2] M. C. Cross and P. C. Hohenberg, Rev. Mod. Phys. 65, 851 (1993).

[3] L. A. Lugiato and R. Lefever, Phys. Rev. Lett. 58, 2209 (1987).

[4] W. J. Firth, A. J. Scroggie, G. S. McDonald, and L. Lugiato, Phys. Rev. A 46, R3609 (1992).

[5] J. B. Geddes, J. V. Moloney, E. M. Wright, and W. J. Firth, Opt. Commun. 111, 623 (1994).

[6] M. Hoyuelos, P. Colet, and M. San Miguel, Phys. Rev. E 58, 74 (1998).

[7] M. Hoyuelos, P. Colet, M. San Miguel, and D. Walgraef, Phys. Rev. E 58, 2992 (1998).

[8] R. W. Boyd, Nonlinear Optics (Academic Press, San Diego, 1992).

[9] G. Grynberg, Opt. Commun. 48, 432 (1984).

[10] M. Ducloy and D. Bloch, Phys. Rev. A 30, 3107 (1984).

[11] M. S. Malcuit, D. J. Gauthier, and R. W. Boyd, Opt. Lett. 13, 663 (1988).

[12] In Ref. [5] $I_{0}$ is defined as the intensity of any of the components of the input field, so in the equation equivalent to Eq. (2) in Ref. [5], $I_{0}$ is not divided by 2 .

[13] R. Montagne, E. Hernández-García, A. Amengual, and M. San Miguel, Phys. Rev. E 56, 151 (1997).

[14] D. Walgraef, Spatio-Temporal Pattern Formation (SpringerVerlag, New York, 1996). 